\title{
Entwicklung der interkulturellen Kompetenz anhand von Materialien für den Fremdsprachenunterricht
}

ABSTRACT. The article presents the methodology of foreign language coursebooks evaluation that makes it possible to verify the extent to which coursebook contents and tasks support the development of intercultural competence. The suggested criteria of coursebook analysis comprise many complex aspects that can be divided into four general categories: 1 . the concept of coursebooks, 2 . the relationship between language and culture, 3 . intercultural competence in the process of learning and teaching a foreign language, 4. teachers as the target group of coursebooks users.

Im Zuge der Internationalisierung vieler wirtschaftlicher, sozialer und kultureller Bereiche sowie persönlicher Mobilität stellt sich die Frage, ob und inwieweit die polnische Schule Kinder und Jugendliche auf die Ansprüche des Lebens in einer solchen Wirklichkeit der kulturellen und sprachlichen Vielfalt vorbereiten kann. Die Entwicklung der interkulturellen Kompetenz nimmt dabei eine zentrale Stellung ein.

Der vorliegende Beitrag möchte einen Einblick in die Möglichkeiten der Förderung dieser Kompetenz im Rahmen des DaF-Unterrichts liefern. Der Schwerpunkt dieser Ausführungen liegt in dem Einsatz von Unterrichtsmaterialien. Die Wahl dieses Aspektes ist damit zu begründen, dass die Unterrichtsmaterialien zweifelsohne die Inhalte des didaktischen Geschehens sowie den Umgang mit ihnen bestimmen. Bezüglich der interkulturellen Dimension ist das von besonderer Relevanz, denn je nachdem, welcher Unterrichtsmaterialien sich die Lehrkräfte bedienen, werden die Lernenden nur mit der Fremdsprache an sich oder auch mit der weit aufgefassten Kultur des neuen / anderen Sprachraumes vertraut gemacht. 
Das in diesem Artikel vorgeschlagene Instrumentarium zur Analyse von Unterrichtsmaterialien umfasst mehrere Fragenkomplexe, die um vier Aspekte gruppiert werden: I. Konzeption der Unterrichtsmaterialien, II. Verhältnis von Sprache und Kultur, III. Interkulturelle Kompetenz im DaFUnterricht, IV. Lehrkräfte als Zielgruppe der Unterrichtsmaterialien.

Im Folgenden werden diese erläutert - zunächst in tabellarischer Form als ein Kriterienraster und dann ergänzend als Besprechung der jeweiligen Fragenkomplexe.

\section{Konzeption der Unterrichtsmaterialien (Kriterien 1 und 2)}

Kriterium 1 - Bestandteile des Kursmaterials (KM)

- Aus welchen Elementen setzt sich das KM zusammen? (Lehrbuch, Schülerheft, Lehrerhandreichungen, Folien, CDs, Kassetten etc.)

- Welche Elemente werden als Basis- und welche als Zusatzkomponenten vorgesehen?

- Werden Defizite in einzelnen Komponenten durch die anderen ausgeglichen?

Kriterium 2 - Didaktische Konzeption der Autoren / Autorinnen

- Wie sieht das grundlegende Konzept des KM aus?

- Ist das KM „universalistisch” oder regional konzipiert?

Bei der Untersuchung sollten die Qualität und der Inhalt aller zu jedem Titel vorgesehenen Komponenten, soweit sie vorhanden sind, beachtet werden. Dies ist notwendig, weil nur auf diese Art und Weise das Gesamtbild des Kursmaterials ermittelt werden kann. Die für das interkulturelle Lernen wesentlichen Bausteine in Form bestimmter Inhalten, Aufgaben, Übungen, Anregungen u. Ä. können in verschiedenen Bestandteilen des Kursmaterials (z.B. auf den Tonträgern, in Handreichungen oder Kopiervorlagen etc.) präsent sein. In dem Raster wird der Terminus Kursmaterial (KM - diese Abkürzung ist als alle möglichen Deklinationsformen zu verstehen) als Benennung eines konkreten Beispiels der Unterrichtsmaterialien, eines konkreten Titels, anhand dessen ein konkreter Kurs gestaltet werden kann, verwendet.

Zu Beginn der Analyse sollten je nach Möglichkeit Angaben zur Konzeption mit einbezogen werden, falls diese den Lehrerhandreichungen oder einem anderen Teil der Materialien zu entnehmen sind. Diese Vorgehensweise ist insofern von Bedeutung, als dass in der Evaluation außer den extrinsischen Kriterien (den in dem hier vorgestellten Raster) ebenfalls die didaktischen Absichten der Autoren und Autorinnen berücksichtigt werden sollten, um im Sinne der intrinsischen Analyse überprüfen zu können, inwieweit diese ihrem eigenen Anspruch gerecht werden.

In der interkulturellen Didaktik spielt die Orientierung des Unterrichtskonzepts am Lernenden und an der Lernergruppe eine bedeutende Rolle. Möchte man im Hinblick darauf den Deutschunterricht in den der Zielspra- 
che fernen und kulturell unterschiedlichen Ländern planen sowie gestalten und dabei diese Adressatenorientierung nicht aus den Augen verlieren, so sollte das Fremdsprachenlernen an der Umwelt und den praktischen Erfahrungen der Lernenden angesetzt werden. Solchen Forderungen können die im deutschen Sprachraum entstandenen und für den weltweiten Einsatz gedachten (d.h. "universalistischen" und adressatenunspezifischen) Unterrichtsmaterialien nur in einem sehr begrenzten Umfang oder gar nicht gerecht werden. Soweit beim Entwerfen der Unterrichtsmaterialien nämlich keine sprachlich und kulturell näher bestimmte rezipierende Gruppe ins Auge gefasst wird, ist eine genauere Betrachtung und Einbeziehung der Bedingungen von Sprach- und Kulturvermittlung unmöglich. Deswegen können solche Unterrichtsmaterialien den besonderen Erfordernissen des Unterrichts vor Ort nicht entsprechen (vgl. Götze 1996).

Regionale Lehrwerke sind in diesem Zusammenhang als solche zu verstehen, die auf der Basis der im deutschsprachigen Raum entwickelten Lehrwerke von einem deutsch-einheimischen Autorenteam partnerschaftlich erarbeitet werden. Sie sollen den speziellen Bedürfnissen, den besonderen Lerntraditionen und Lerngewohnheiten, den bildungspolitischen Voraussetzungen, dem Stand der Lehrerausbildung sowie den curricularen Rahmenbedingungen des Landes oder der Region angepasst werden. Nicht gemeint sind hier demnach Lehrwerke, die in der Tradition einheimischer Lehrwerkgestaltung von einheimischen Autoren und Autorinnen verfasst und im Land verlegt werden. (Breitung / Lattaro 2001: 1043)

\section{Verhältnis von Sprache und Kultur (Kriterium 3)}

Kriterium 3 - Kulturbegriff

- Bildet die Kulturvermittlung einen integralen Teil der Sprachvermittlung im Prozess des Fremdsprachenlernens? Woran lässt sich das erkennen?

- Welcher Rang wird der Kultur von den Autoren / Autorinnen in der Konzeption des KM zugeschrieben?

- Wird Kultur in dem KM als Bündel verschiedener miteinander verknüpfter Bereiche verstanden? Welche Aspekte sind gewählt worden? Gibt es eine Begründung für das Vorhandensein dieser Bereiche z.B. in Lehrerhandreichungen?

- Wird der Systemcharakter der Kultur berücksichtigt? Gibt es Elemente, die der Oberflächenstruktur oder/und der Tiefenstruktur zugeordnet werden können? Gibt es Hinweise diesbezüglich für Lehrende?

- Wird die kulturelle Vielfalt der heutigen Wirklichkeit in die Konzeption des KM einbezogen? Werden die vielfältigen Relationen der Kulturen zueinander angesprochen?

In der Analyse muss zuerst davon angegangen werden, ob in den Materialien kulturelle Elemente überhaupt berücksichtigt sind. Ohne solche Elemente kann der Prozess des interkulturellen Lernens gar nicht zustande kommen. Es soll also zu Stande (im Neuschwick) festgestellt werden, ob die 
Unterrichtsmaterialien in den fremdsprachlichen Lernprozessen die Begegnung mit der fremden Kultur ermöglichen oder ob sie sich lediglich auf die Vermittlung der Fremdsprache und Förderung der Sprachfertigkeiten konzentrieren.

Kultur ist nicht angeboren sondern wird erworben (vgl. Herskovits 1940: 625). Hinsichtlich der anderen, fremden Kulturen findet dieser Erwerb vorwiegend im schulischen Rahmen statt, meistens in dem des Fremdsprachenlernens. Der Beirat Deutsch als Fremdsprache des Goethe-Instituts hat diesbezüglich 1992 wertvolle Empfehlungen geliefert: „Die Begegnung mit der fremden Kultur beginnt in der ersten Stunde des Sprachunterrichts. Von daher ist eine Abtrennung der Spracharbeit von der Kultur nicht angemessen. Vielmehr muss die Verschränkung von Kultur und Sprache Konsequenzen haben für die Entwicklung von Vermittlungsmethoden, Lernkonzepten sowie für Lehrmaterialien" (Beirat DaF 1992: 112-113).

Kultur beinhaltet verschiedene miteinander verknüpfte und voneinander abhängige Bereiche, wie z.B. Sprache, Geographie, Geschichte, Wirtschaft, Politik, Literatur, Kunst, Wissenschaft, Technik, Wertvorstellungen, Verhaltensmuster, Religion sowie Sozialstrukturen (vgl. Keller 1996: 228). Daraus ergibt sich, dass der Fremdsprachenunterricht sich nicht dem Anspruch stellen sollte, das Ganze einer fremden Kultur zu erfassen und abzubilden. Vielmehr geht es darum, nur die Elemente zu wählen, die - je nach den individuellen Möglichkeiten der Lehrenden und Lernenden auf jeweiliger Stufe sowie mit Berücksichtigung der Lern- und Lehrziele - am meisten zur "Einübung in das Fremdverstehen" (Christ 1996: 1) beitragen können.

Kultur ist mit einem System vergleichbar. Laut der Systemtheorie weisen alle Systeme eine Oberflächenstruktur und eine Tiefenstruktur auf. In Bezug auf Kultur sind im Rahmen der Oberflächenstruktur formale Elemente wie beispielsweise Artefakte, Institutionen sowie die in ihnen vollzogenen Rituale zu nennen. Auf der Seite der inhaltlichen Tiefenstruktur befinden sich hingegen Wertorientierungen, Identitätsmuster, Denk-, Empfindungs- und Wahrnehmungsweisen (vgl. Keller 1994: 81-85). Dieser Systemcharakter der Kultur ist für das Fremdsprachenlernen und -lehren von Relevanz. Er verdeutlicht, dass die alleinige Kenntnis der formalen Oberflächenstruktur ohne Aufspüren der inhaltlichen Steuerungsmechanismen des Handelns, die in den Tiefenstrukturen der jeweiligen Kultur verborgen sind, keinen Zugang zum Verständnis der anderen Kultur gewährleisten.

Entsprechend diesen Überlegungen gilt es zu überprüfen, wie die Kultur in den Unterrichtsmaterialien verstanden wird, wie weiterhin die vorhandenen kulturellen Erscheinungen dargestellt werden und ob der Systemcharakter der Kultur berücksichtigt wird. Darüber hinaus soll nach einer Antwort auf die Frage gesucht werden, ob die vielfältigen Beziehungen der 
Kulturen zueinander thematisiert werden und auf welche Art und Weise dies laut der Konzeption der Unterrichtsmaterialien geschieht.

\section{Interkulturelle Kompetenz im DaF-Unterricht (Kriterien 4 bis 9)}

\section{Kriterium 4 - Soziokulturelles Wissen - konkrete Themenbereiche}

- Welche Merkmale des soziokulturellen Wissens aus dem Referenzrahmen sind im $\mathrm{KM}$ präsent?

- Anhand welcher Mittel (Texte, Bilder etc.) werden diese Inhalte vorgestellt?

- In welchen Komponenten des KM befinden sie sich? Nur im Lehrbuch? Auch als (notwendige?) Ergänzung im Arbeitsbuch? In Lehrerhandreichungen (gibt es hier kulturelle Zusatzinformationen für Lehrkräfte?)?

Kriterium 5 - Visualisierungselemente als Informationsträger

- Welche Arten von Visualisierungselementen kommen in dem KM vor? Erfüllen sie die ihnen jeweils zugeschriebenen Funktionen?

- Welche Rezeptionsbedingungen stellt das Bildmaterial? (Vorwissen, Vorerfahrungen, sprachliche Voraussetzungen)

- Wie ist die Qualität der Visualisierungselemente (technische Qualität, Bildinhalt, Aktualität, Repräsentativität, Offenheit, Verhältnis zum Text)?

Kriterium 6 - Auseinandersetzung mit der eigenen und der fremden / anderen Kultur

- Berücksichtigen die kulturbezogenen Aspekte sowohl die Ziel- als auch die Ausgangskultur? Falls keine direkte Verbindung der beiden Kulturen deutlich wird: Gibt es Hinweise für mögliche Vernetzungen in Lehrerhandreichungen?

- Wie wird das Eigene und das Fremde dargestellt? Kommt es zu einer Gegenüberstellung beider Ebenen oder können die Lernenden durch die Darstellungsart Einblicke in den relationalen Charakter des Verhältnisses zwischen beiden Ebenen gewinnen?

- Tragen die Inhalte sowie die Darstellungsart des Fremden und des Eigenen zur Entwicklung des Bewusstseins von Gemeinsamkeiten und Unterschieden zwischen der eigenen und fremden Kultur bei?

Kriterium 7 - Wahrnehmungsschulung

- Wird eigenkulturelle / fremdkulturelle Wahrnehmung thematisiert? Sind Hinweise für Lehrkräfte vorhanden? Gibt es explizite Übungen und Aufgaben dazu?

- Gibt es Aufgaben zur Entwicklung der Fähigkeit, im Vertrauten das Fremde und im Fremden das Vertraute zu entdecken?

- Wird den Lernenden bewusst gemacht, dass ihre eigenen sprachlichen und kulturellen Schemata ihre Wahrnehmung und Deutung der fremden Sprache und Kultur beeinflussen?

Kriterium 8 - Perspektivenwechsel / Relativierung von Perspektiven

- Wird die Vielfältigkeit und Mehrschichtigkeit der eigenen und fremden Kultur thematisiert?

- Werden die Themen / Situationen mehrperspektivisch dargestellt?

- Gibt es Situationen, Übungen, die der Relativierung von Perspektiven dienen?

- Gibt es Aufgaben, die Vergleichs- und Identifikationsmöglichkeiten bieten? Wird bei der Erarbeitung sprachlicher und soziokultureller Phänomene vergleichend verfahren? 
- Wird die soziale Sensibilität gefördert? Gibt es Gelegenheiten, in denen die Lernenden Mitgefühl oder Rücksichtnahme entwickeln können?

- Werden Verfahren angeboten, bei denen sich die Lernenden in die Anderen versetzen, ihre Bedürfnisse erkennen und im eigenen Verhalten berücksichtigen können?

- Wird im KM die Perspektive der Autoren / Autorinnen deutlich? Wie kommt sie zur Sprache? Ist das eine andere Perspektive als die der Lernenden?

Kriterium 9 - Vorstellungen und Einstellungen

- Wird das Bild der Zielsprachkultur nur auf die Bundesrepublik Deutschland reduziert oder gibt es Themenbereiche, welche regionale Vielfalt der deutschsprachigen Länder berücksichtigen?

- Anhand welcher Mittel wird das Bild des Landes / der Länder vorgestellt (fiktive Texte, Sachbeschreibungen, dokumentarische Texte, literarische Texte, Fotos etc.)?

- Ist die Darstellung realistisch? Ist das Bild authentisch, aktuell, objektiv?

- Wie repräsentativ sind die gewählten Beispiele, anhand derer die Lernenden Einblicke in die Zielkultur gewinnen sollen?

- Gibt es Verallgemeinerungen und Vereinfachungen? Werden dadurch stereotypische Denkweisen vermittelt?

- Gibt es Aspekte, die mit bestimmten Wertungen vermittelt werden (positive oder negative Darstellung)?

- Werden Vorstellungen und Einstellungen, die die Lernenden bereits in ihrer eigenen Kultur erworben haben, berücksichtigt?

- Wird diese Vorgeprägtheit thematisiert oder bewusst gemacht?

Die Kriterien aus dem Raster zu diesem Aspekt werden in zwei Gruppen gegliedert: 1. Vermittlung des soziokulturellen Wissens (4 und 5), 2. Förderung der interkulturellen Kompetenz in anderen Bereichen (6 bis 9).

Es muss ergänzend zu den Begriffen „das Fremde / das Eigene, fremdkulturelle / eigenkulturelle Elemente, Zielkultur / Ausgangskultur", die relationalen Charakter aufweisen, gesagt werden, dass sie in den Kursmaterialien aus der Sicht der Lernenden zu betrachten sind, d.h., das Fremde für die Lernenden betrifft die neue, fremde (hier: deutsche) Sprache und Kultur, das Eigene wiederum (hier) die polnische Sprache und Kultur.

Interkulturelle Kompetenz wird nicht als Weiterentwicklung der seit den 70er Jahren in der Fremdsprachendidaktik entwickelten Zielsetzung der kommunikativen Kompetenz gesehen, weil sie nicht in erster Linie auf die sprachliche Ebene ausgerichtet ist. Vielmehr gilt sie als "rein affektivverhaltensorientiert" und wird mit Aussagen wie 'die fremde Kultur verstehen', 'zu Toleranz fähig sein', 'ethnozentrische Sichtweisen vermeiden' usw. umschrieben (House 1996: 2). Sie wird somit als zusätzliche Kompetenz (neben der kommunikativen Kompetenz) angesehen, die sich auf kulturelle Elemente in fremdsprachlichen Lern- und Lehrprozessen bezieht.

Dabei muss erwähnt werden, dass in der deutschsprachigen Fachliteratur der letzten Jahre außer der kommunikativen Kompetenz und der interkulturellen Kompetenz auch solche Stimmen gefunden werden können, die 
vom Konzept der interkulturellen kommunikativen Kompetenz sprechen (s. z.B. Röttger 2004). Diese Bezeichnung verbindet die beiden vorher genannten Kompetenzen und weist auf zwei Tatsachen hin - zum einen darauf dass die bisherige Umsetzung des Konzeptes der kommunikativen Kompetenz aufgrund der nur unzureichenden Berücksichtigung der kulturellen Eingebundenheit von Sprache und sprachlichem Handeln ungenügend ist, und zum anderen darauf, dass nur dieser Terminus die Trennung von Sprach- und Kulturvermittlung im FU aufheben kann. (Röttger 2004: 44).

In der Realität zeigt sich ein oft "nur" kommunikativ ausgerichteter FU, in dem die Kommunikation in der fremden Sprache keinen bewusst wahrgenommenen interkulturellen Charakter aufweist. Würde man statt der kommunikativen Kompetenz die interkulturelle kommunikative Kompetenz zur Zielsetzung des FUs erklären, so müsste folglich die interkulturelle Dimension des FUs viel stärker ins Blickfeld rücken. Entsprechend sollten bei der Gestaltung des FUs die beiden sich aufeinander beziehenden Kompetenzen - nämlich die kommunikative Kompetenz und die interkulturelle Kompetenz - wenn auch nicht zu einer Einheit verschmolzen, so doch als integrale Bestandteile verstanden werden.

In dem vorliegenden Beitrag wird diese Zweiteilung der Kompetenzen beibehalten. Dies resultiert vor allem daraus, dass der Schwerpunkt der Analyse der Unterrichtsmaterialien in der interkulturellen Kompetenz liegt und folglich die Aspekte der kommunikativen Kompetenz (z.B. die Entwicklung der Sprachfertigkeiten oder Vermittlung der Grammatik) nicht untersucht werden.

Unter Leitung einer Expertengruppe des Europarates ist ein "Gemeinsamer europäischer Referenzrahmen für Sprachen" (Europarat 2001) verfasst worden. Dieses Kompendium für das Lernen, Lehren und Beurteilen von Kenntnissen in fremden Sprachen stellt alle wichtigen Bereiche der Lehr- und Lernprozesse (wie z.B. Ziele, Methoden, Fertigkeitsstufen und Verfahren der Evaluation) dar. Deshalb werden die Hinweise und Feststellungen dieser Publikation als Ausgangspunkt zur Betrachtung über die interkulturelle Kompetenz genutzt.

Im Referenzrahmen werden , allgemeine Kompetenzen' von ,kommunikativen Sprachkompetenzen' unterschieden. An die zuvor dargestellte Zielsetzung des Fremdsprachenlernens anknüpfend, zeigen sich Elemente der interkulturellen Kompetenz in der ersten Gruppe - der allgemeinen Kompetenzen - wieder, während die zweite Gruppe Aspekte der kommunikativen Kompetenz umfasst.

$\mathrm{Zu}$ den Bestandteilen der interkulturellen Kompetenz gehören (Europarat 2001: 104-106):

1. im Bereich des Wissens (Kenntnisse, die gespeichert und abgerufen werden können):

- Soziokulturelles Wissen (Das ist Wissen über die Gesellschaft und die Kultur der Gemeinschaft oder der Gemeinschaften, in denen eine 
Sprache gesprochen wird. Es verdient besondere Aufmerksamkeit, weil es sehr wahrscheinlich außerhalb des früheren Erfahrungsbereichs des Lernenden liegt und zudem durch Stereotypen verzerrt sein kann.)

Merkmale des soziokulturellen Wissens können sich auf das tägliche Leben, Lebensbedingungen, interpersonale Beziehungen, Werte, Überzeugungen und Einstellungen, Körpersprache, soziale Konventionen sowie rituelles Verhalten beziehen.

- Interkulturelles Bewusstsein Es erwächst aus der Kenntnis, dem Bewusstsein und dem Verständnis der Beziehungen zwischen der "Welt des Herkunftslandes" und der "Welt der Zielsprachengemeinschaft" (Ähnlichkeiten und klare Unterschiede). Es bedeutet auch, sich der regionalen und sozialen Verschiedenheit in beiden Welten bewusst zu sein. Ferner wird es durch das Bewusstsein bereichert, dass es noch viele andere Kulturen gibt, was dazu verhilft, beide Kulturen in einen größeren Kontext einzuordnen.

Über das objektive Wissen hinaus gehört zu diesem Teil der interkulturellen Kompetenz auch, dass man sich bewusst ist, wie eine Gemeinschaft jeweils aus der Perspektive der anderen erscheint, nämlich häufig in Form nationaler Stereotype.)

2. im Bereich des Könnens (Umsetzung des Wissens in Routinehandlungen):

- Interkulturelle Fertigkeiten, die umfassen: 1. die Fähigkeit, die Ausgangskultur und die fremde Kultur miteinander in Beziehung zu setzen; 2. kulturelle Sensibilität und die Fähigkeit, eine Reihe verschiedener Strategien für den Kontakt mit Angehörigen anderer Kulturen zu identifizieren und zu verwenden; 3. die Fähigkeit, als kultureller Mittler zwischen der eigenen und der fremden Kultur zu agieren und wirksam mit interkulturellen Missverständnissen und Konfliktsituationen umzugehen; 4. die Fähigkeit, stereotype Beziehungen zu überwinden.

Durch Beantwortung der Fragenkomplexe zu diesem Aspekt soll unter anderem erfahren werden, inwieweit die angebotenen Inhalte die fremdund eigenkulturellen Themen betreffen und welche konkreten Informationen es sind, sowie ob die Aufgaben den Lernenden eine positive Öffnung für das Andere / Fremde ermöglichen und sie zum interkulturellen Denken anregen.

Einen bedeutenden Gesichtspunkt stellt hier die Wahrnehmungsschulung dar. Wer in Kontakt mit einer anderen / fremden Kultur tritt, muss sich bewusst werden, dass Wahrnehmung selektiv ist und von persönlichen, gruppenspezifischen und kulturspezifischen Interessen, Erfahrungen etc. 
geleitet ist. Deswegen ist es äußerst wichtig, die eigene Wahrnehmung kontrollieren $\mathrm{zu}$ lernen, d.h. erkennen $\mathrm{zu}$ können, dass die eigenkulturellen Wahrnehmungskriterien fremde Situationen verformen. Ohne diese Fähigkeit kommt man kaum zu adäquaten Interpretationen und Einschätzungen der Gegebenheiten. Wahrnehmung ist aktive Interpretation, die ständig vollzogen wird, damit die Welt verständlich erscheint. Sie schließt fast immer Wertung ein, wobei das Fremde / Andere, Ungewohnte oft eher negativ beurteilt wird, weil die Funktionen und der Sinnzusammenhang nicht bekannt sind (Bachmann u.a. 1995: 10-12, Simon-Pelanda 2001: 940).

Ein ebenfalls wichtiges Gebiet stellt die Förderung der Fähigkeit zum Perspektivenwechsel dar, d.h. der Fähigkeit, sich in die Lage des Anderen einzufühlen, seine Bedürfnisse $z u$ erkennen und im eigenen Verhalten $\mathrm{zu}$ berücksichtigen, die Wahrnehmung des Gesprächspartners zu übernehmen, aus der eigenen Rolle in die eines Anderen zu wechseln. Sie ist notwendig, um eine andere Kultur zu verstehen. (vgl. Schinschke 1995; Bredella / Christ 2000).

Der Erfolg der Schulung dieser Fähigkeit mithilfe von Unterrichtsmaterialien ist davon abhängig, ob sie differenziert vorgetragene, perspektivisch vermittelte Inhalte und dazu entsprechende Aufgaben anbieten. $\mathrm{Zu}$ dieser Mehrperspektivität gehört als erstes die Berücksichtigung der Einstellungen und Vorstellungen, die die Lernenden bereits in ihrer eigenen Kultur erworben haben. Es muss ihnen bewusst gemacht werden, dass sie diese Vorgeprägtheit besitzt. Es muss ihnen auch deutlich gemacht werden, dass Vergleichen eine Methode der Erkenntnisgewinnung ist und dass zum Verständnis des Anderen / Fremden ein Perspektivenwechsel nötig ist, dass sie quasi in die Haut des Anderen / Fremden schlüpfen müssen, um ihn verstehen zu können (vgl. Bachmann u.a. 1995; Abendroth-Timmer 1999; Macaire / Hosch 2000). Bemerkenswert ist an dieser Stelle, dass sich die deutschen Verhältnisse oder auch weiter gesehen die Verhältnisse im deutschsprachigen Raum oft für vielfältige und differenzierte Darstellungsweisen eignen und dadurch auch einen intrakulturellen Vergleich ermöglichen. Das erlaubt den Lernenden wiederum einen Vergleich mit den Verhältnissen im eigenen Land, im Rahmen eines interkulturellen Vergleichs.

Als zweites müssen die fremdkulturellen Inhalte / Situationen selbst mehrperspektivisch dargestellt werden - aus der Sicht verschiedener Rollen, sozialer Positionen, historischer Stellungen usw. (vgl. Abendroth-Timmer 2000). Es muss den Lernenden bewusst werden, dass die Mitteilung eines Textes nur eine unter vielen möglichen ist.

Abhängig von der Provenienz der Unterrichtsmaterialien zeigen sich Unterschiede in der Widerspiegelung bzw. Konstruktion der Realität des zielsprachlichen Landes. So wird i.d.R. bei den im deutschen Sprachraum 
entstandenen Materialien die Innenperspektive eingenommen, während in den von Nichtdeutschsprachigen außerhalb des deutschen Sprachraums entwickelten Materialien die Außenperspektive gebraucht wird. (vgl. Macaire / Hosch 2000).

Eben dadurch, dass im FU immer mehrere Perspektiven zu Wort kommen, können bei den Lernenden Stereotypenbildungen vermieden werden. Das Ergebnis des Vergleichsprozesses kulturell verschieden geprägter $\mathrm{Au}$ ßen- und Innenperspektiven sind vielfältige Brechungen und Bezüge: zwischen dem Eigenen und dem jeweils konkreten Fremden sowie dem Eigenen und dem Fremden im Ich des Verstehenden (Bredella / Christ 2000: XXI).

Vorstellungen, Bilder, Images sind Begriffe, die man oft mit Ländern, Kulturen und derer Vertretern in Verbindung bringt. Manchmal sind sie recht vage und allgemein, manchmal aber sehr detailliert. Als subjektive Gebilde, die nur in den Köpfen der Beteiligten existieren, können sie mehr oder weniger richtig, aber auch über objektive Sachverhalte nur wenig oder nichts aussagen und falsch sein. Doch sie leisten dem Menschen eine Orientierungshilfe in seiner Lebenswelt und wirken sich im Verhalten so aus, als wären sie objektiv zutreffend. Diese Vorstellungen werden i.d.R. mit bestimmten Einstellungen (Meinungen, Attitüden, Überzeugungen, Vorurteilen) verbunden, d.h. mit wertenden Stellungnahmen zu Personen, Gruppen, Sachverhalten, Objekten etc. Bei Begegnungen von Vertretern verschiedener Kulturen sind auf beiden Seiten fast immer solche Vorstellungen und Einstellungen vorhanden und beeinflussen den Begegnungsprozess. Sie hängen eng miteinander zusammen und sind oft nur schwerpunktmäßig voneinander zu trennen. Bei Vorstellungen liegt der Akzent auf dem kognitiven, bei Einstellungen auf dem affektiven Bereich. (vgl. Maletzke 1996: 116-120, Mitulla 1997: 62-78).

Der Auseinandersetzung mit diesen beiden Kategorien (Einstellungen und Vorstellungen) wird im FU, vor allem eben im Rahmen des interkulturellen Lernens, große Bedeutung zugemessen. In der Diskussion um Stereotypen und Vorurteile (vgl. Zawadzka 1997) wird viel häufiger gefordert, sie abzubauen als sich mit ihnen eingehender $z u$ befassen, d.h. sie "kontrastiv und vergleichend $\mathrm{zu}$ beschreiben und zu diskutieren, also zum echten Gegenstand des FUs zu machen" (vgl. Kaikkonen 1990: 232). Dabei existieren sie nun einmal und sind als notwendige psychologische Instrumente zur Erfassung der Wirklichkeit aus dem alltäglichen Leben nicht wegzudenken. Die Forderung nach ihrem Abbau bedeutet wohl gerade den falschen Weg zu einem besseren Verstehen vom Eigenen und Anderen / Fremden, wenn man sich zunächst nicht sorgfältig mit diesen Formen der Vorstellungen und Einstellungen (sowohl mit den eigenen als auch mit fremden) auseinander setzt. Vorurteile können als eine "Schwelle" bezeichnet werden, „die man 
überspringen zu müssen glaubt". Man sollte hingegen auf ihr „lange genug verweilen, denn sie ist der Punkt, an dem beide Kulturen aufeinander treffen" (Steinmann 1992: 219). Mit der Behandlung von Stereotypen und Vorurteilen, ihrer Bewusstwerdung sowie eventuell mit ihrer Relativierung wird das Begreifen des Anderen in seiner Komplexität gefördert. (vgl. Doyé 1993, Ertelt-Vieth 1993).

\section{Lehrkräfte als Zielgruppe der Unterrichtsmaterialien (Kriterium 10)}

Kriterium 10 - Lehrkräfte

- Welches soziokulturelle Wissen und welche interkulturellen Fähigkeiten werden als Vorraussetzung für die Durchführung des didaktisch-erzieherischen Prozesses gesehen?

- Gibt es Hinweise, Zusatzinformationen oder sonstige Hilfestellungen für Lehrkräfte, die keine ausreichende interkulturelle Kompetenz in den FU mitbringen?

Da Lehrkräfte sich in der Praxis von den Unterrichtsmaterialien möglichst große Unterstützung erhoffen, soll das letzte Kriterium in dem vorgestellten Raster zur Analyse der Unterrichtsmaterialien Auskunft darüber geben, welche Ansprüche an die Lehrkräfte selbst gestellt werden, die anhand solcher Materialien interkulturelle Lehr-/Lernprozesse gestalten möchten. Die Suche nach Antworten auf die beiden Fragen dieses Kriteriums sollten die Lehrkräfte zur Selbstreflexion über die eigene interkulturelle Kompetenz bewegen. Gerade diese entscheidet sehr oft darüber, ob die Möglichkeit zur Durchführung von interkulturellen Lern-/Lehrprozessen und somit auch der Entwicklung der interkulturellen Kompetenz der Lernenden anhand dieser Kursmaterialien besteht.

Der vorliegende Beitrag beschäftigt sich mit nur einem Gesichtspunkt, der die Förderung der interkulturellen Kompetenz betrifft. Dies muss aber in einem größeren Kontext gesehen werden. Die Einstellung der Lehrkräfte zum Fremdsprachenlernen überhaupt, ihre erwähnte interkulturelle Kompetenz, die ihnen zur Verfügung stehende Zeit für den Kontakt der Lernenden mit der fremden Sprache in der Schule und die Erwartungen der Eltern bezüglich des fremdsprachlichen Leistungszuwachses ihrer Kinder sollten als mögliche Einflussgrößen nicht aus den Augen verloren werden.

\section{LITERATURVERZEICHNIS}

Abendroth-Timmer, D., 1999, Interkulturelles Lernen und das Bedingungsgefüge der Lehrwerkerstellung. In: W. Bömer/K. Vogel (Hrsg.), Lehrwerke im Fremdsprachenunterricht. Bochum: AKS-Verlag: 166-190.

Abendroth-Timmer, D., 2000, Lernziel „interkulturelle Kompetenz" oder: Wie zeitgemäß sind unsere Lehrwerke?. In: R. Fery/V. Raddatz (Hrsg.), Lehrwerke und ihre Alternativen. Frankfurt/M.: Lang: 35-45. 
Bachmann, S. u.a., 1995, Sichtwechsel NEU 1, 2, 3: Allgemeine Einführung. München: Klett.

Beirat DaF des Goethe-Instituts, 1992, 25 Thesen zur Sprach- und Kuiturvermittlung im Ausland', In: Zielsprache Deutsch 23/2: 112-113.

Bredella, L./Christ, H., (Hrsg.), 2000, Wie ist Fremdverstehen lehr- und lernbar? Tübingen: Narr.

Breitung, H., Lattaro, E. 2001, Regionale Lehrwerke und Lehrmethoden, In: G. Helbig/ L. Götze/G. Henrici/H.-J. Krumm (Hrsg.), Deutsch als Fremdsprache. Ein Internationales Handbuch. Berlin: Walter de Gruyter: 1041-1053.

Christ, H. 1996. Fremdverstehen und interkulturelles Lernen, In: Zeitschrift für interkulturellen Fremdsprachenunterricht (online). 1 (3).

Doyé, P., 1993, Fremdsprachenunterricht ohne Stereotypen? Sozialpsychologische, logische und pädagogische Aspekte. In: J.-P. Timm/H.J. Vollmer (Hrsg.), Kontroversen in der Fremdsprachenforschung. Bochum: Brockmeyer: 408-416.

Ertelt-Vieth, A. 1993. Abbauen, Tolerieren, Betonen? Stereotype zwischen Vorurteil und Kulturspezifik. In: J.-P. Timm/H.J. Vollmer (Hrsg.), Kontroversen in der Fremdsprachenforschung. Bochum: Brockmeyer: 425-433.

Europarat. Rat für kulturelle Zusammenarbeit, 2001, Gemeinsamer europäischer Referenzrahmen für Sprachen: lernen, lehren, beurteilen. Berlin, München: Langenscheidt.

Götze, L., 1996, Grundsätze für die Erstellung regionaler Lehrwerke. In: B. Kast/G. Neuner, Zur Analyse, Begutachtung und Entwicklung von Lehrwerken für den fremdsprachlichen Deutschunterricht. Berlin, München: Langenscheidt: 243-246.

Herskovits, M., 1940, Man and His Works. The Science of Cultural Anthropology. New York: Alfred A. Knopf.

House, J., 1996, Zum Erwerb Interkultureller Kompetenz im Unterricht des DaF, In: Zeitschrift für interkulturellen Fremdsprachenunterricht (online). 1 (3).

Kaikkonen, P., 1990, Interkulturelle Kultur- und Landeskunde und interkultureller FU. In: Neusprachliche Mitteilungen aus Wissenschaft und Praxis 1990/4: 230-236.

Keller, G. 1994. Interkulturelles Lernen aus der Perspektive von Systemtheorie und Sozialpsychologie. In: Neusprachliche Mitteilungen aus Wissenschaft und Praxis 1994/2: 81-85.

Keller, G., 1996., Zehn Thesen zur Neuorientierung des interkulturellen Lernens. In: Praxis des neusprachlichen Unterrichts, 1996/3: 227-236.

Macaire, D./Hosch, W., 2000, Bilder in der Landeskunde. Berlin / München: Langenscheidt.

Maletzke, G., 1996, Interkulturelle Kommunikation: zur Interaktion zwischen Menschen verschiedener Kulturen. Opladen: Westdeutscher Verlag.

Mitulla, C., 1997, Die Barriere im Kopf. Stereotype und Vorurteile bei Kindern gegenüber Ausländern. Opladen: Leske und Budrich.

Röttger, E., 2004, Interkulturelles Lernen im Fremdsprachenunterricht. Hamburg: Kovač.

Schinschke, A., 1995, Perspektivenübernahme als grundlegende Fähigkeit im Umgang mit Fremden. In: L. Bredella, H. Christ (Hrsg.), Didaktik des Fremdverstehens. Tübingen: Narr: 36-50.

Simon-Pelanda, H., 2001, Landeskundliches Lernen und Lehren. In: G. Helbig/L. Götze/ G. Henrici/H.-J. Krumm (Hrsg.), Deutsch als Fremdsprache. Ein Internationales Handbuch. Berlin: Walter de Gruyter, 931-942.

Steinmann, S., 1992, Vorurteile? Ja, bitte! Plädoyer für den redlichen Umgang mit Vorurteilen im Fremdsprachenunterricht. In: Zielsprache Deutsch 23/4: 217-224.

Zawadzka, E. 1997 Stereotype und Vorurteile im FU. Sollte man sie bekämpfen, akzeptieren oder relativieren?. In: Glottodidactica, Vol. XXV: 121-133. 